\title{
Pacific
}

Journal of

Mathematics

\section{ON GENERALIZED WEIGHTED HILBERT MATRICES}

Emmanuel Preissmann and Olivier LÉvêQue

Volume 265 No. 1 


\title{
ON GENERALIZED WEIGHTED HILBERT MATRICES
}

\author{
EMmanuel PREISSMANN AND OliVIER LÉVÊQUE
}

We study spectral properties of generalized weighted Hilbert matrices. In particular, we establish results on the spectral norm, the determinant, and various relations between the eigenvalues and eigenvectors of such matrices. We also study the asymptotic behavior of the spectral norm of the classical Hilbert matrix.

\section{Introduction}

The classical infinite Hilbert matrices

(1) $T_{\infty}=\left(\begin{array}{cccccc}\ddots & \ddots & \ddots & \ddots & \ddots & \ddots \\ \ddots & 0 & -1 & -\frac{1}{2} & -\frac{1}{3} & \ddots \\ \ddots & 1 & 0 & -1 & -\frac{1}{2} & \ddots \\ \ddots & \frac{1}{2} & 1 & 0 & -1 & \ddots \\ \ddots & \frac{1}{3} & \frac{1}{2} & 1 & 0 & \ddots \\ \ddots & \ddots & \ddots & \ddots & \ddots & \ddots\end{array}\right) \quad$ and $\quad H_{\infty}=\left(\begin{array}{ccccc}1 & \frac{1}{2} & \frac{1}{3} & \frac{1}{4} & \ldots \\ \frac{1}{2} & \frac{1}{3} & \frac{1}{4} & \frac{1}{5} & \ddots \\ \frac{1}{3} & \frac{1}{4} & \frac{1}{5} & \frac{1}{6} & \ddots \\ \frac{1}{4} & \frac{1}{5} & \frac{1}{6} & \frac{1}{7} & \ddots \\ \vdots & \ddots & \ddots & \ddots & \ddots\end{array}\right)$

have been widely studied in the mathematical literature, for a variety of good reasons (see [Choi 1983] for a nice survey of their astonishing properties). In this paper, we present results and conjectures on spectral properties of these matrices and related types of matrices. We first review known results in Section 2, and then introduce new results in Section 3 on generalized weighted Hilbert matrices of the form

$$
b_{m, n}(\boldsymbol{x}, \boldsymbol{c})=\left\{\begin{array}{cl}
0 & \text { if } m=n, \\
\frac{c_{m} c_{n}}{x_{m}-x_{n}} & \text { if } m \neq n .
\end{array}\right.
$$

Our results can be summarized as follows. Theorem 1 states a surprising property of these matrices: Their spectral norm depends monotonically on the absolute values of their entries, a property known a priori only for matrices with positive entries. Theorem 2 says that the determinants of such matrices are polynomials in the square

MSC2010: 26D15.

Keywords: Hilbert matrices, Hilbert inequalities, eigenvalue-eigenvector relations. 
of their entries. In Lemma 5, we prove a key relation between the eigenvalues and eigenvectors of these matrices, which leads to a chain of nice consequences, including Corollaries 1 and 2. Our work finds its roots in [Montgomery and Vaughan 1973], a seminal paper that initiated the study of generalized Hilbert matrices.

Notation. Let $p>1$. In what follows, $\|\boldsymbol{y}\|_{p}$ denotes the $\ell^{p}$-norm of the vector $\boldsymbol{y} \in \mathbb{C}^{S}:$

$$
\|\boldsymbol{y}\|_{p}:=\left(\sum_{k=1}^{S}\left|y_{k}\right|^{p}\right)^{1 / p} .
$$

For an $S \times S$ matrix $M,\|M\|_{p}$ denotes the matrix norm induced by this vector norm:

$$
\|M\|_{p}:=\sup _{\|\boldsymbol{y}\|_{p}=1}\|M \boldsymbol{y}\|_{p} .
$$

In the particular case $p=2$, the following simplified notation will be adopted:

$$
\|\boldsymbol{y}\|_{2}=\|\boldsymbol{y}\| \text { (Euclidean norm) and }\|M\|_{2}=\|M\| .
$$

When $M$ is normal (i.e., when $M M^{*}=M^{*} M$, where $M^{*}$ stands for the complexconjugate transpose of the matrix $M$ ), the above norm is equal to the spectral norm of $M$ :

$$
\|M\|=\sup \{|\lambda|: \lambda \in \operatorname{Spec}(M)\}
$$

\section{A survey of classical results and conjectures}

2.1. Hilbert's inequalities. The infinite-dimensional matrices presented in (1) are two different versions of the classical Hilbert matrix. Notice first that $T_{\infty}$ is a Toeplitz matrix (i.e., a matrix whose entry $n, m$ depends only on the difference $m-n$ ), while $H_{\infty}$ is a Hankel matrix (i.e., a matrix whose entry $n, m$ depends only on the sum $n+m$ ). The Hilbert inequalities state (see [Hardy et al. 1952, p. 212]) that

$$
\left|\sum_{m, n \in \mathbb{Z}} u_{m}\left(T_{\infty}\right)_{m, n} v_{n}\right| \leq \pi \quad \text { for } \boldsymbol{u}, \boldsymbol{v} \in \ell^{2}(\mathbb{Z} ; \mathbb{C}) \text { with }\|\boldsymbol{u}\|=\|\boldsymbol{v}\|=1
$$

and

$$
\left|\sum_{m, n \in \mathbb{N}} u_{m}\left(H_{\infty}\right)_{m, n} v_{n}\right| \leq \pi \quad \text { for } \boldsymbol{u}, \boldsymbol{v} \in \ell^{2}(\mathbb{N} ; \mathbb{C}) \text { with }\|\boldsymbol{u}\|=\|\boldsymbol{v}\|=1 ;
$$

here $\pi$ cannot be replaced by a smaller constant. ${ }^{1}$ This is saying that $T_{\infty}$ and $H_{\infty}$ are bounded operators in $\ell^{2}(\mathbb{Z} ; \mathbb{C})$ and $\ell^{2}(\mathbb{N} ; \mathbb{C})$, respectively, with norm $\pi$.

Titchmarsh [1926] proved that $\left\|T_{\infty}\right\|_{p}<\infty$. Hardy, Littlewood and Pólya [1952,

\footnotetext{
${ }^{1}$ Hilbert originally proved these inequalities with $2 \pi$ instead of $\pi$; the optimal constant was found later by Schur.
} 
p. 227] obtained the explicit expression

$$
\left\|H_{\infty}\right\|_{p}=\frac{\pi}{\sin (\pi / p)} \text { for all } p>1
$$

It is clear that $\left\|T_{\infty}\right\|_{p} \geq\left\|H_{\infty}\right\|_{p}$, as $H_{\infty}$ may be seen as the lower left corner of $T_{\infty}$ (up to a column permutation), but no exact value is known for it (except in the case where $p=2^{n}$ or $p=2^{n} /\left(2^{n}-1\right)$ for some integer $n \geq 1$; see [Laeng 2007; 2009] for a review of the subject).

Consider the corresponding $R \times R$ matrices $T_{R}$ and $H_{R}$, defined by

$$
\left(T_{R}\right)_{m, n}=\left\{\begin{array}{cl}
0 & \text { if } m=n, \\
\frac{1}{m-n} & \text { if } m \neq n,
\end{array} \quad\left(H_{R}\right)_{m, n}=\frac{1}{m+n-1} \quad \text { for } 1<m, n<R .\right.
$$

The Hilbert inequalities imply that for every integer $R \geq 1$,

$$
\left\|T_{R}\right\|<\pi \text { and }\left\|H_{R}\right\|<\pi \text {. }
$$

Clearly also $\left\|T_{R}\right\|$ and $\left\|H_{R}\right\|$ increase as $R$ increases, and

$$
\lim _{R \rightarrow \infty}\left\|T_{R}\right\|=\lim _{R \rightarrow \infty}\left\|H_{R}\right\|=\pi .
$$

A question of interest is the convergence speed of $\left\|H_{R}\right\|$ and $\left\|T_{R}\right\|$ toward their common limiting value $\pi$. Up to a column permutation, $H_{R}$ can be seen as the lower left corner of $T_{2 R+1}$, so $\left\|H_{R}\right\| \leq\left\|T_{2 R+1}\right\|$ for every integer $R \geq 1$. This hints at a slower convergence speed for the matrices $H_{R}$ than for the matrices $T_{R}$. Indeed, Wilf and de Bruijn (see [Wilf 1970]) have shown that

$$
\pi-\left\|H_{R}\right\| \sim \frac{\pi^{5}}{2(\log R)^{2}} \quad \text { as } R \rightarrow \infty,
$$

whereas there exist $a, b>0$ such that

$$
\frac{a}{R}<\pi-\left\|T_{R}\right\|<\frac{b \log R}{R} \quad \text { for } R \geq 2 .
$$

We will prove these inequalities at the end of this paper. The lower bound has been proved by Montgomery (see [Matthews 2002]), and it has been conjectured in [Preissmann 1985], and independently by Montgomery, that the upper bound in the previous inequality is tight, i.e., that

$$
\pi-\left\|T_{R}\right\| \sim \frac{c \log R}{R} \quad \text { as } R \rightarrow \infty .
$$

We also provide some numerical evidence for this conjecture at the end of the paper. 
2.2. Toeplitz matrices and Grenander-Szegó's theorem. We review the theory developed by Grenander and Szegő [1958] to analyze the asymptotic spectrum of Toeplitz matrices. In particular, we cite their result on the convergence speed of the spectral norm of such matrices.

Let $\left(c_{r}\right)_{r \in \mathbb{Z}}$ be a sequence of complex numbers such that

$$
\sum_{r \in \mathbb{Z}}\left|c_{r}\right|<\infty
$$

and let us define the corresponding function, or symbol:

$$
f(x)=\sum_{r \in \mathbb{Z}} c_{r} \exp (\operatorname{ir} x) \quad \text { for } x \in[0,2 \pi] .
$$

Because of the assumption made on the Fourier coefficients $c_{r}$, the function $f$ is continuous, and of course $f(0)=f(2 \pi)$. Equivalently, $f$ can be viewed as a continuous $2 \pi$-periodic function on $\mathbb{R}$.

Now let $C_{R}$ be the $R \times R$ matrix defined by

$$
\left(C_{R}\right)_{m, n}=c_{m-n} \quad \text { for } 1 \leq m \text { and } n \leq R .
$$

One checks by direct computation that, for any vector $\boldsymbol{u} \in \mathbb{C}^{R}$ with $\|\boldsymbol{u}\|^{2}:=$ $\sum_{1 \leq n \leq R}\left|u_{n}\right|^{2}=1$, we have

$$
\boldsymbol{u}^{*} C_{R} \boldsymbol{u}=\int_{0}^{2 \pi} f(x)|\phi(x)|^{2} d x
$$

where

$$
\phi(x)=\frac{1}{\sqrt{2 \pi}} \sum_{1 \leq n \leq R} u_{n} \exp (i(n-1) x) .
$$

Let us now assume that $C_{R}$ is a normal matrix $\left(C_{R} C_{R}^{*}=C_{R}^{*} C_{R}\right)$; this is the case, for example, when $f$ is a real-valued function (in which case $C_{R}$ is Hermitian: $\left.C_{R}^{*}=C_{R}\right)$. As $\|\boldsymbol{u}\|=1$, we also have $\int_{0}^{2 \pi}|\phi(x)|^{2} d x=1$, which implies that

$$
\left\|C_{R}\right\| \leq \sup _{x \in[0,2 \pi]}|f(x)|=: M
$$

for any integer $R \geq 1$. Grenander and Szegó [1958, p. 72] proved the following refined statement on the convergence speed of the spectral norm. If $f$ is twice continuously differentiable, admits a unique maximum in $x_{0}$ and is such that $f^{\prime \prime}\left(x_{0}\right) \neq 0$, then

$$
M-\left\|C_{R}\right\| \sim f\left(x_{0}\right)-f\left(x_{0}+\frac{\pi}{R}\right) \sim \frac{\pi^{2}\left|f^{\prime \prime}\left(x_{0}\right)\right|}{2 R^{2}} \quad \text { as } R \rightarrow \infty .
$$

This result does not apply to Hilbert matrices of the form $T_{R}$ : Since the harmonic 
series diverges, condition (5) is not satisfied. Correspondingly, the symbol associated with these matrices is the function

$$
f(x)=\sum_{r \geq 1} \frac{-\exp (i r x)+\exp (-i r x)}{r}=-2 i \sum_{r \geq 1} \frac{\sin (r x)}{r}=i(x-\pi)
$$

for $x \in$ ]0, $2 \pi$ [, while by Dirichlet's theorem $f(0)=f(2 \pi)=0$. The function $f$ is therefore discontinuous, but relation (6) still holds in this case and allows us to deduce Hilbert's inequality:

$$
\left\|T_{R}\right\| \leq \sup _{x \in[0,2 \pi]}|f(x)|=\pi .
$$

However, relation (6) alone does not allow us to draw conclusions on the convergence speed toward $\pi$.

Evaluating the convergence speed of the spectral norm is a difficult problem when $f$ attains its maximum at a point of discontinuity. An interesting matrix of this type was studied in detail in [Slepian 1978]; ${ }^{2}$ known as the prolate matrix, it is defined as

$$
\left(P_{R}\right)_{m, n}=p_{m-n} \quad \text { for } 1 \leq m \text { and } n \leq R, \quad \text { where } p_{r}=\left\{\begin{array}{cl}
\frac{\sin (2 \pi w r)}{r} & \text { if } r \neq 0, \\
2 \pi w & \text { if } r=0,
\end{array}\right.
$$

for $0<w<\frac{1}{2}$ a fixed parameter. Here, again, we see that condition (5) is not satisfied. The symbol associated with this matrix is the function

$$
\begin{aligned}
f_{w}(x) & =\sum_{r \in \mathbb{Z}} p_{r} \exp (i r x)=2 \pi w+2 \sum_{r \geq 1} \frac{\sin (2 \pi w r)}{r} \cos (r x) \\
& =\pi 1_{[0,2 \pi w] \cup[2 \pi(1-w), 2 \pi]}(x)
\end{aligned}
$$

for all $x \in[0,2 \pi] \backslash\{2 \pi w, 2 \pi(1-w)\}$. In this case, we again have for any integer $R \geq 1$

$$
\left\|P_{R}\right\|<\sup _{x \in[0,2 \pi]}\left|f_{w}(x)\right|=\pi \quad \text { and } \quad \lim _{R \rightarrow \infty}\left\|P_{R}\right\|=\pi .
$$

It is moreover shown in [Slepian 1978] that for all $0<\omega<\frac{1}{2}$, there exist $c_{w}, d_{w}>0$ (given explicitly in [Varah 1993]) such that

$$
\pi-\left\|P_{R}\right\| \sim c_{w} \sqrt{R} \exp \left(-d_{w} R\right) .
$$

We see here that although the function $f_{w}$ is discontinuous, the convergence speed is exponential, not polynomial (as is the case with a smooth symbol). Of course, the

${ }^{2}$ See also [Varah 1993] for a recent exposition of the problem; we are thankful to Ben Adcock for pointing out this interesting reference to us. 
situation here is quite particular, as the function $f_{w}$ has a plateau at its maximum value, which is not the case for the Hilbert matrix $T_{R}$.

2.3. Generalized weighted Hilbert matrices. Let $\boldsymbol{x}=\left(x_{1}, \ldots, x_{R}\right)$ be a vector of distinct real numbers and $\boldsymbol{c}=\left(c_{1}, \ldots, c_{R}\right)$ any vector of real numbers. We define the $R \times R$ matrix $B(\boldsymbol{x}, \boldsymbol{c})$ by formula (2). We also set

$$
A(\boldsymbol{x})=B(\boldsymbol{x}, \mathbf{1}), \quad \text { where } \mathbf{1}=(1, \ldots, 1) .
$$

If there is no risk of confusion, we write $A$ and $B$ instead of $A(\boldsymbol{x})$ and $B(\boldsymbol{x}, \boldsymbol{c})$.

Thus $A(\boldsymbol{x})$ is the classical Hilbert matrix. To motivate the study of the generalization $B(\boldsymbol{x}, \boldsymbol{c})$, we mention that Montgomery and Vaughan [1973] proved that

and that

$$
\|A(\boldsymbol{x})\| \leq \frac{\pi}{\delta}, \quad \text { with } \delta=\inf _{\substack{1 \leq m, n \leq R \\ m \neq n}}\left|x_{n}-x_{m}\right|,
$$

$$
\|B(\boldsymbol{x}, \boldsymbol{c})\| \leq \frac{3 \pi}{2}, \quad \text { with } c_{n}=\sqrt{\min _{\substack{1 \leq m \leq R \\ m \neq n}}\left|x_{m}-x_{n}\right| .}
$$

They also conjectured that the tightest upper bound is $\|B(\boldsymbol{x}, \boldsymbol{c})\| \leq \pi$. Montgomery and Vaughan's result was improved in [Preissmann 1984] to $\|B(\boldsymbol{x}, \boldsymbol{c})\| \leq 4 \pi / 3$, but the conjecture remains open so far.

We conclude this section with some applications.

Large sieve inequalities. Suppose the real numbers $x_{1}, \ldots, x_{R}$ are distinct modulo 1 . Let $\|t\|$ denote the distance from a real number $t$ to the closest integer, and let

$$
\delta:=\min _{r, s, r \neq s}\left\|x_{r}-x_{s}\right\| \quad \text { and } \quad \delta_{r}:=\min _{s, s \neq r}\left\|x_{r}-x_{s}\right\| .
$$

For an arbitrary sequence of complex numbers $\left(a_{n}\right)_{M+1 \leq n \leq M+N}$, we write

$$
S(x):=\sum_{M+1 \leq n \leq M+N} a_{n} \exp (2 \pi i n x) .
$$

A large sieve inequality has the generic form

$$
\sum_{1 \leq r \leq R}\left|S\left(x_{r}\right)\right|^{2} \leq \Delta(N, \delta) \sum_{M+1 \leq n \leq M+N}\left|a_{n}\right|^{2} .
$$

Using Hilbert's inequality (3), one can show that the previous inequality holds with $\Delta(N, \delta)=N+\delta^{-1}-1$. Equivalently, this says that if

$$
B:=\left\{\exp \left(2 \pi i n x_{r}\right)\right\}_{M+1 \leq n \leq M+N, 1 \leq r \leq R}
$$

then

$$
\|B\|^{2} \leq \Delta(N, \delta)
$$


Generalized Hilbert inequalities of type (8) are particularly useful when studying irregularly spaced $x_{r}$ (such as Farey sequences), as they allow us to prove the following refined large sieve inequality:

$$
\sum_{1 \leq r \leq R}\left(N+\frac{3}{2} \delta_{r}^{-1}\right)^{-1}\left|S\left(x_{r}\right)\right|^{2} \leq \sum_{M+1 \leq n \leq M+N}\left|a_{n}\right|^{2} .
$$

This last result is useful for arithmetic applications. It allows us to show, for example, that $\pi(M+N)-\pi(M) \leq 2 \pi(N)$, where $\pi(N)$ is the number of primes smaller than or equal to $N$ (see [Montgomery and Vaughan 1973]). By contrast, the inequality $\pi(M+N)-\pi(M) \leq \pi(N)$ stands as a conjecture so far.

The Bombieri-Vinogradov theorem, which is related to various conjectures on the distribution of primes, is another important application of large sieve inequalities (see [Bombieri et al. 1986], for instance).

Other Hilbert inequalities. Montgomery and Vaughan [1974] studied variants of Hilbert's inequality (with, for instance, $1 /\left(x_{r}-x_{s}\right)$ replaced by $\left.\csc \left(x_{r}-x_{s}\right)\right)$, which allow them to show that if $\sum_{n \geq 1} n\left|a_{n}\right|^{2}<\infty$, then

$$
\int_{0}^{T}\left|\sum_{n \geq 1} a_{n} n^{-i t}\right|^{2} d t=\sum_{n \geq 1}\left|a_{n}\right|^{2}(T+O(n)) .
$$

The key idea behind the proof of the main result in their paper is the identity

$$
\csc \left(x_{k}-x_{l}\right) \csc \left(x_{l}-x_{m}\right)=\csc \left(x_{k}-x_{m}\right)\left(\cot \left(x_{k}-x_{l}\right)+\cot \left(x_{l}-x_{m}\right)\right),
$$

which is of the same type as our relation (10) below. A further generalization of Hilbert's inequalities has been built on this in [Preissmann 1987], where we solved the functional equations

and

$$
\frac{1}{\theta(x) \theta(y)}=\Psi(x)-\Psi(y)+\frac{\phi(x-y)}{\theta(x-y)}
$$

$$
\frac{1}{\theta(x) \theta(y)}=\frac{\sigma(x)-\sigma(y)}{\theta(x-y)}+\tau(x) \tau(y) \quad \text { with } \tau(0)=0 .
$$

\section{New results}

3.1. Spectral norm of $\boldsymbol{B}(\boldsymbol{x}, \boldsymbol{c})$. In this subsection we state and prove our first main result, on the monotonicity of the spectral norm of matrices $B(\boldsymbol{x}, \boldsymbol{c})$.

Theorem 1. If $\boldsymbol{x}, \boldsymbol{x}^{\prime}, \boldsymbol{c}$ and $\boldsymbol{c}^{\prime}$ are vectors of real numbers such that

$$
\left|b_{m, n}(\boldsymbol{x}, \boldsymbol{c})\right| \leq\left|b_{m, n}\left(\boldsymbol{x}^{\prime}, \boldsymbol{c}^{\prime}\right)\right| \quad \text { for } 1 \leq m \text { and } n \leq R,
$$

then

$$
\|B(\boldsymbol{x}, \boldsymbol{c})\| \leq\left\|B\left(\boldsymbol{x}^{\prime}, \boldsymbol{c}^{\prime}\right)\right\|
$$


Remark. For matrices $Y$ and $Z$ with positive entries, if $0 \leq y_{m, n} \leq z_{m, n}$ for all $m$ and $n$, then $\|Y\| \leq\|Z\|$. Indeed, consider the normalized eigenvector $\boldsymbol{u}$ corresponding to the largest eigenvalue of $Y^{*} Y$ : Since $Y^{*} Y$ has positive entries, $\boldsymbol{u}$ is also positive, so $\|Y\|=\|Y \boldsymbol{u}\| \leq\|Z \boldsymbol{u}\| \leq\|Z\|$. The above result states that a similar result holds for matrices of the form $B(\boldsymbol{x}, \boldsymbol{c})$, even though these do not have positive entries.

We decompose the proof of Theorem 1 into a sequence of lemmas. We will use several times the relation

$$
a_{k, l} a_{l, m}=a_{k, m}\left(a_{k, l}+a_{l, m}\right) \text { for } k, l, m \text { distinct, }
$$

where $a_{m, n}=1 /\left(x_{m}-x_{n}\right)$.

Lemma 1. If $k$ is a positive integer and $1 \leq n \leq R$, then, denoting by $B_{-n}$ the matrix $B$ with the $n$-th row and column removed, we have

$$
S:=\sum_{\substack{1 \leq l, m \leq R \\ l \neq n, m \neq n, l \neq m}} b_{n, l} b_{m, n}\left(B_{-n}^{k}\right)_{l, m}=0 .
$$

Proof. Using (10), we obtain

$$
\begin{aligned}
S & =\sum_{\substack{1 \leq l, m \leq R \\
l \neq n, m \neq n, l \neq m}} c_{l} c_{m} c_{n}^{2} a_{m, n} a_{n, l}\left(B_{-n}^{k}\right)_{l, m} \\
& =\sum_{\substack{1 \leq l, m \leq R \\
l \neq n, m \neq n, l \neq m}} c_{l} c_{m} c_{n}^{2} a_{m, l}\left(a_{m, n}+a_{n, l}\right)\left(B_{-n}^{k}\right)_{l, m}=: S_{1}+S_{2},
\end{aligned}
$$

where

$$
\begin{aligned}
S_{1} & =\sum_{\substack{1 \leq l, m \leq R \\
l \neq n, m \neq n, l \neq m}} c_{l} c_{m} c_{n}^{2} a_{m, l} a_{m, n}\left(B_{-n}^{k}\right)_{l, m} \\
& =\sum_{\substack{1 \leq l, m \leq R \\
l \neq n, m \neq n, l \neq m}} c_{n}^{2} b_{m, l} a_{m, n}\left(B_{-n}^{k}\right)_{l, m}=\sum_{\substack{1 \leq m \leq R \\
m \neq n}} c_{n}^{2} a_{m, n}\left(B_{-n}^{k+1}\right)_{m, m}
\end{aligned}
$$

and

$$
\begin{aligned}
S_{2} & =\sum_{\substack{1 \leq l, m \leq R \\
l \neq n, m \neq n, l \neq m}} c_{l} c_{m} c_{n}^{2} a_{m, l} a_{n, l}\left(B_{-n}^{k}\right)_{l, m} \\
& =\sum_{\substack{1 \leq l \leq R \\
l \neq n}} c_{n}^{2} a_{n, l}\left(B_{-n}^{k+1}\right)_{l, l}=-\sum_{\substack{1 \leq l \leq R \\
l \neq n}} c_{n}^{2} a_{l, n}\left(B_{-n}^{k+1}\right)_{l, l}=-S_{1},
\end{aligned}
$$

since $A$ is antisymmetric. 
Lemma 2. Let $1 \leq n \leq R$ and $k \geq 2$ be an integer. Then

$$
\begin{aligned}
\left(B^{k}\right)_{n, n} & =\sum_{0 \leq r \leq k-2} \sum_{\substack{1 \leq l, m \leq R \\
l \neq n, m \neq n}} b_{n, l}\left(B_{-n}^{r}\right)_{l, m} b_{m, n}\left(B^{k-r-2}\right)_{n, n} \\
& =-\sum_{0 \leq r \leq k-2} \sum_{1 \leq l \leq R} b_{n, l}^{2}\left(B_{-n}^{r}\right)_{l, l}\left(B^{k-r-2}\right)_{n, n} .
\end{aligned}
$$

Proof. Notice first that

$$
\left(B^{k}\right)_{n, n}=\sum_{1 \leq n_{1}, \ldots, n_{k-1} \leq R} b_{n, n_{1}} b_{n_{1}, n_{2}} \ldots b_{n_{k-2}, n_{k-1}} b_{n_{k-1}, n} .
$$

As $b_{n, n}=0$, we may consider $n_{1}, n_{k-1} \neq n$ in this sum. For each $\left(n_{1}, \ldots, n_{k-1}\right)$, define

$$
s=\inf \left\{t \in\{2, \ldots, k\} \mid n_{1} \neq n, \ldots, n_{t-1} \neq n, n_{t}=n\right\},
$$

where, by convention, $n_{k}=n$. Ordering the terms in the above sum according to the value of $s$, we obtain

$$
\begin{aligned}
\left(B^{k}\right)_{n, n} & =\sum_{2 \leq s \leq k} \sum_{n_{1}, n_{s-1} \neq n} b_{n, n_{1}}\left(B_{-n}^{s-2}\right)_{n_{1}, n_{s-1}} b_{n_{s-1}, n}\left(B^{k-s}\right)_{n, n} \\
& =\sum_{0 \leq r \leq k-2} \sum_{n_{1}, n_{r+1} \neq n} b_{n, n_{1}}\left(B_{-n}^{r}\right)_{n_{1}, n_{r+1}} b_{n_{r+1}, n}\left(B^{k-r-2}\right)_{n, n},
\end{aligned}
$$

which is the first equality in the lemma. The second follows from (11) and the fact that $B$ is antisymmetric.

Lemma 3. Let $1 \leq n \leq R$ and let $k \geq 2$ be an integer.

- If $k$ is odd, then $\left(B^{k}\right)_{n, n}=0$.

- If $k$ is even, then $(-1)^{k / 2}\left(B^{k}\right)_{n, n}$ is a polynomial in the $b_{l, m}^{2}, 1 \leq l<m \leq R$, with positive coefficients.

Proof. Since $B$ is antisymmetric, the first statement is obvious. The second follows by induction from Lemma 2 .

Proof of Theorem 1. Observe that since the matrix $i B$ is Hermitian, it has $R$ real eigenvalues $\mu_{1}, \ldots, \mu_{R}$ corresponding to an orthonormal basis of eigenvectors, so

$$
\|B\|=\max _{1 \leq r \leq R}\left|\mu_{r}\right| .
$$

And for a positive integer $k$, we have

$$
\operatorname{Tr}\left(B^{2 k}\right)=\sum_{1 \leq r \leq R}(-1)^{k} \mu_{r}^{2 k}
$$


Therefore, we obtain

$$
\|B\|=\lim _{k \rightarrow \infty}\left((-1)^{k} \operatorname{Tr}\left(B^{2 k}\right)\right)^{1 / 2 k},
$$

and the theorem follows from Lemma 3.

3.2. Determinant of $\boldsymbol{B}(\boldsymbol{x}, \boldsymbol{c})$. Our next result shows that the determinant of $B(\boldsymbol{x}, \boldsymbol{c})$ is a polynomial in the $b_{l, m}^{2}$.

Theorem 2. If $R$ is odd, then $\operatorname{det}(B(\boldsymbol{x}, \boldsymbol{c}))=0$. If $R=2 T$ is even, then

$$
\operatorname{det}(B(\boldsymbol{x}, \boldsymbol{c}))=\prod_{k=1}^{R} c_{k}^{2} \sum_{\left(m_{i}, n_{i}\right)_{1}^{T} \in E} \prod_{i=1}^{T} a_{m_{i}, n_{i}}^{2}=\sum_{\left(m_{i}, n_{i}\right)_{1}^{T} \in E} \prod_{i=1}^{T} b_{m_{i}, n_{i}}^{2},
$$

where

$$
E:=\left\{\left(m_{i}, n_{i}\right)_{1}^{T} \mid \bigcup_{i=1}^{T}\left\{m_{i}, n_{i}\right\}=\{1, \ldots, R\}, m_{i}<n_{i} \text { for all } i, \text { and } m_{1}<\cdots<m_{T}\right\} .
$$

Lemma 4. Let $l$ be an integer, with $3 \leq l \leq R$. Denoting by $\mathscr{S}_{l}$ the set of permutations of $\{1, \ldots, l\}$, we have

$$
S:=\sum_{\sigma \in \mathscr{Y}_{l}} a_{\sigma(1), \sigma(2)} a_{\sigma(2), \sigma(3)} \ldots a_{\sigma(l-1), \sigma(l)} a_{\sigma(l), \sigma(1)}=0 .
$$

Proof. We define

$$
\begin{aligned}
S_{1} & :=\sum_{\sigma \in \mathscr{Y}_{l}} a_{\sigma(1), \sigma(2)} a_{\sigma(2), \sigma(3)} \ldots a_{\sigma(l-1), \sigma(1)} a_{\sigma(l-1), \sigma(l)}, \\
S_{2} & :=\sum_{\sigma \in \mathscr{Y}_{l}} a_{\sigma(1), \sigma(2)} a_{\sigma(2), \sigma(3)} \ldots a_{\sigma(l-1), \sigma(1)} a_{\sigma(l), \sigma(1)} .
\end{aligned}
$$

By (10), we have $S=S_{1}+S_{2}$. Now let $\tau \in \mathscr{Y}_{l}$ be the permutation defined by $\tau(1)=l-1, \tau(2)=1, \tau(3)=2, \ldots, \tau(l-1)=l-2, \tau(l)=l$. We obtain

$$
\begin{aligned}
S_{2} & =\sum_{\sigma \in \mathscr{S}_{l}} a_{\sigma \tau(1), \sigma \tau(2)} a_{\sigma \tau(2), \sigma \tau(3)} \ldots a_{\sigma \tau(l-1), \sigma \tau(1)} a_{\sigma \tau(l), \sigma \tau(1)} \\
& =\sum_{\sigma \in S_{l}} a_{\sigma(l-1), \sigma(1)} a_{\sigma(1), \sigma(2)} \ldots a_{\sigma(l-2), \sigma(l-1)} a_{\sigma(l), \sigma(l-1)}=-S_{1},
\end{aligned}
$$

which completes the proof.

Proof of Theorem 2. By definition,

$$
\operatorname{det}(B)=\sum_{\sigma \in \mathscr{S}_{R}} \varepsilon(\sigma) \prod_{1 \leq n \leq R} a_{n, \sigma(n)} c_{n}^{2} .
$$

Every permutation $\sigma$ is a product of $k$ cycles, with $1 \leq k \leq n$. We denote by $F_{1}, \ldots, F_{k}$ the supports of these cycles and by $n_{1}, n_{2}, \ldots, n_{k}$ their cardinalities, and we set 


$$
S\left(F_{i}\right):=\frac{1}{n_{i}} \sum_{s_{1}, s_{2}, \ldots, s_{n_{i}} \mid\left\{s_{1}, s_{2}, \ldots, s_{n_{i}}\right\}=F_{i}} a_{s_{1}, s_{2}} a_{s_{2}, s_{3}} \ldots a_{s_{n_{i}-1}, s_{n_{i}}} a_{s_{n_{i}}, s_{1}} .
$$

In the above expression for $\operatorname{det}(B)$, the contribution of the permutations having $F_{1}, \ldots, F_{k}$ as supports for their cycles is of the

$$
(-1)^{n_{1}+n_{2}+\cdots+n_{k}-k} \prod_{i=1}^{k} S\left(F_{i}\right) \prod_{r=1}^{R} c_{r}^{2} .
$$

Hence, by (13) and the fact that the main diagonal is zero, a nonzero contribution can only occur when all cycles are of cardinality 2 , which proves the theorem.

Remark. The above statement allows us to recover part of the conclusion of Lemma 3. First notice that by Theorem 2 and for all $J \subset\{1, \ldots, R\}, \operatorname{det}\left(B_{J}\right)$, where $B_{J}=\left(b_{l, m}\right)_{l, m \in J}$, is also a polynomial in the $b_{l, m}^{2}$. Define

$$
\sigma_{k}=\sum_{\substack{J \subset\{1, \ldots, R\} \\|J|=k}} \prod_{\substack{i \in J\\}} \lambda_{i}
$$

where $\lambda_{1}, \ldots, \lambda_{R}$ are the eigenvalues of $B$. Notice that

$$
\sigma_{k}=\sum_{\substack{J \subset\{1, \ldots, R\} \\|J|=k}} \operatorname{det}\left(B_{J}\right)
$$

Indeed, let $P$ be the polynomial defined as $P(x)=\prod_{1 \leq i \leq R}\left(x-\lambda_{i}\right)$. We observe that, on one hand, the matrix-valued version of this polynomial is given by

$$
P(x)=\prod_{1 \leq i \leq R}\left(x-\lambda_{i} I\right)=x^{R}+\sum_{k=1}^{R} x^{R-k}(-1)^{k} \sum_{\substack{J \subset\{1, \ldots, R\} \\|J|=k}} \prod_{i \in J} \lambda_{i}=x^{R}+\sum_{1 \leq k \leq R} x^{R-k}(-1)^{k} \sigma_{k},
$$

while, on the other hand,

$$
P(x)=\prod_{i=1}^{R}\left(x-\lambda_{i}\right)=\operatorname{det}(x I-B)=x^{R}+\sum_{k=1}^{R} x^{R-k}(-1)^{k} \sum_{\substack{J \subset\{1, \ldots, R\} \\|J|=k}} \operatorname{det}\left(B_{J}\right),
$$

so by identifying the coefficients we obtain equality (14). This implies that $\sigma_{k}$ is also a polynomial in the $b_{l, m}^{2}$. Finally, for $s_{l}=\sum_{1 \leq i \leq R} \lambda_{i}^{l}$, we have the following recursion, also known as Newton-Girard's formula:

$$
s_{l}=\sum_{1 \leq i \leq l-1}(-1)^{i-1} \sigma_{i} s_{l-i}+(-1)^{l-1} l \sigma_{l} .
$$

For example, $s_{0}=n, \quad s_{1}=\sigma_{1}, \quad s_{2}=s_{1} \sigma_{1}-2 \sigma_{2}, \quad s_{3}=s_{2} \sigma_{1}-s_{1} \sigma_{2}+3 \sigma_{3}$, etc. We therefore find by induction that for all $k,(-1)^{k} \operatorname{Tr}\left(B^{2 k}\right)=(-1)^{k} s_{2 k}$ is also 
a polynomial in the $b_{l, m}^{2}$, but this alone does not guarantee the positivity of the coefficients obtained in Lemma 3 above.

3.3. Formulas regarding the eigenvalues and eigenvectors of $A(x)$ and $B(x, c)$. We first state the following lemma, which has important consequences for the eigenvalues of the matrices $A(\boldsymbol{x})$ and $B(\boldsymbol{x}, \boldsymbol{c})$, as we will see. The approach taken below generalizes the method initiated by Montgomery and Vaughan [1973].

Lemma 5. (a) Let $\boldsymbol{u}=\left(u_{1}, \ldots, u_{R}\right)^{T}$ be an eigenvector of $A(\boldsymbol{x})$ for the eigenvalue $i \mu$. Then for $1 \leq n \leq R$, we have

$$
\mu^{2}\left|u_{n}\right|^{2}=\sum_{1 \leq m \leq R} a_{m, n}^{2}\left(\left|u_{m}\right|^{2}+2 \Re\left(u_{n} \bar{u}_{m}\right)\right) .
$$

(b) Let $\boldsymbol{u}=\left(u_{1}, \ldots, u_{R}\right)^{T}$ be an eigenvector of $B(\boldsymbol{x}, \boldsymbol{c})$ for the eigenvalue $i \mu$. Then for $1 \leq n \leq R$, we have

$$
\mu^{2}\left|u_{n}\right|^{2}=\sum_{1 \leq m \leq R} a_{m, n}^{2}\left(c_{n}^{2} c_{m}^{2}\left|u_{m}\right|^{2}+2 c_{n}^{3} c_{m} \Re\left(u_{n} \bar{u}_{m}\right)\right) .
$$

Proof. We prove (16), from which (15) follows by specializing to the case $\boldsymbol{c}=\mathbf{1}$.

Our starting assumption is $B \boldsymbol{u}=i \mu \boldsymbol{u}$, i.e., $\sum_{1 \leq m \leq R} b_{n, m} u_{m}=i \mu u_{n}$. Taking the modulus square on both sides, we obtain

$$
\mu^{2}\left|u_{n}\right|^{2}=\sum_{\substack{1 \leq l, m \leq R \\ l \neq n, m \neq n}} b_{n, m} b_{n, l} u_{m} \bar{u}_{l}
$$

(Notice that the sum can be taken over $l \neq n$ and $m \neq n$, as $b_{n, n}=0$.) Therefore,

$$
\mu^{2}\left|u_{n}\right|^{2}=c_{n}^{2} \sum_{\substack{1 \leq l, m \leq R \\ l \neq n, m \neq n}} c_{l} c_{m} a_{n, m} a_{n, l} u_{m} \bar{u}_{l}=c_{n}^{2}\left(S_{1}+S_{2}\right),
$$

where $S_{1}$ corresponds to the terms in the sum with $l=m$ and $S_{2}$ is its complement:

$$
S_{1}=\sum_{\substack{1 \leq m \leq R \\ m \neq n}} c_{m}^{2} a_{m, n}^{2}\left|u_{m}\right|^{2}, \quad S_{2}=\sum_{\substack{1 \leq l, m \leq R \\ l \neq m, l \neq n, m \neq n}} c_{l} c_{m} a_{n, m} a_{n, l} u_{m} \bar{u}_{l} .
$$

As $l, m$, and $n$ are all distinct in this last sum, we can use (10) and the antisymmetry of $A$ to obtain

$$
a_{n, m} a_{n, l}=a_{l, m} a_{n, l}+a_{m, l} a_{n, m},
$$

so

$$
S_{2}=\sum_{\substack{1 \leq l, m \leq R \\ l \neq m, l \neq n, m \neq n}} c_{l} c_{m}\left(a_{l, m} a_{n, l}+a_{m, l} a_{n, m}\right) u_{m} \bar{u}_{l}=S_{3}+S_{4}
$$


with

$$
\begin{aligned}
S_{3} & =\sum_{\substack{1 \leq l, m \leq R \\
l \neq m, l \neq n, m \neq n}} c_{l} c_{m} a_{l, m} a_{n, l} u_{m} \bar{u}_{l} \\
& =\sum_{\substack{1 \leq l, m \leq R \\
l \neq m, l \neq n, m \neq n}} b_{l, m} a_{n, l} u_{m} \bar{u}_{l}=\sum_{\substack{1 \leq l \leq R \\
l \neq n}} a_{n, l} \bar{u}_{l} \sum_{\substack{1 \leq m \leq R \\
m \neq l, m \neq n}} b_{l, m} u_{m} .
\end{aligned}
$$

As $\boldsymbol{u}$ is an eigenvector of $B$, it follows that

$$
S_{3}=\sum_{\substack{1 \leq l \leq R \\ l \neq n}} a_{n, l} \bar{u}_{l}\left(i \mu u_{l}-b_{l, n} u_{n}\right)
$$

Likewise, noticing that $\overline{\boldsymbol{u}}$ is also an eigenvector of $B$ (with the corresponding eigenvalue $-i \mu$ ), we obtain

$$
S_{4}=\sum_{\substack{1 \leq m \leq R \\ m \neq n}} a_{n, m} u_{m} \sum_{\substack{1 \leq l \leq R \\ l \neq n}} b_{m, l} \bar{u}_{l}=\sum_{\substack{1 \leq m \leq R \\ m \neq n}} a_{n, m} u_{m}\left(-i \mu \bar{u}_{m}-b_{m, n} \bar{u}_{n}\right) .
$$

From (19), we deduce that

$$
S_{2}=S_{3}+S_{4}=-\sum_{\substack{1 \leq m \leq R \\ m \neq n}} a_{n, m} b_{m, n}\left(\bar{u}_{m} u_{n}+u_{m} \bar{u}_{n}\right)=2 \sum_{\substack{1 \leq m \leq R \\ m \neq n}} a_{m, n} b_{m, n} \Re\left(u_{m} \bar{u}_{n}\right) .
$$

Now, using this together with (17) and (18), we finally obtain

$$
\mu^{2}\left|u_{n}\right|^{2}=\sum_{\substack{1 \leq m \leq R \\ m \neq n}} c_{n}^{2}\left(c_{m}^{2} a_{m, n}^{2}\left|u_{m}\right|^{2}+2 c_{m} c_{n} a_{m, n}^{2} \Re\left(u_{m} \bar{u}_{n}\right)\right)
$$

which completes the proof.

One of the many consequences of Lemma 5 is the following.

Corollary 1. If $c_{1}, \ldots, c_{R}$ are all nonzero, then the eigenvalues of $B(\boldsymbol{x}, \boldsymbol{c})$ are all distinct.

Proof. If in the basis of eigenvectors of $B$ there were two corresponding to the same eigenvalue, it would be possible to find a linear combination of them (also an eigenvector) such that one component (say, $u_{n}$ ) would be equal to zero. Then by (16) we would have

$$
\sum_{1 \leq m \leq R} a_{m, n}^{2} c_{n}^{2} c_{m}^{2}\left|u_{m}\right|^{2}=0
$$

which is impossible, given the assumption made.

A more precise version of Lemma 5(b) reads as follows. 
Lemma 6. Let $\boldsymbol{u}=\boldsymbol{v}+i \boldsymbol{w}\left(\boldsymbol{v}, \boldsymbol{w} \in \mathbb{R}^{R}\right)$ be an eigenvector of $-i \mu$ corresponding to the eigenvalue $B(\boldsymbol{x}, \boldsymbol{c})$. Then

$$
\mu^{2} v_{n}^{2}=\sum_{1 \leq m \leq R} b_{n, m}^{2} w_{m}^{2}+2 c_{n}^{2} \sum_{\substack{1 \leq m \leq R \\ m \neq n}} a_{n, m} w_{m}\left(\mu v_{m}-b_{m, n} w_{n}\right) .
$$

Moreover, if $\mu \neq 0$, then $\|\boldsymbol{v}\|=\|\boldsymbol{w}\|$, while if $\mu=0$, then $\operatorname{det}(B)=0$, so one of the eigenvectors corresponding to this eigenvalue is real.

Proof. Applying the proof method of Lemma 5 gives $\mu^{2} v_{n}^{2}=\left(\sum_{1 \leq m \leq R} b_{n, m} w_{m}\right)^{2}=\sum_{1 \leq m \leq R} b_{n, m}^{2} w_{m}^{2}+\sum_{\substack{1 \leq l, m \leq R \\ l \neq m}} b_{n, m} b_{n, l} w_{m} w_{l}=: S_{1}+S_{2}$.

We can write

with

$$
S_{2}=c_{n}^{2} \sum_{\substack{1 \leq l, m \leq R \\ l \neq m}} c_{l} c_{m} a_{n, m} a_{n, l} w_{m} w_{l}=c_{n}^{2}\left(S_{3}+S_{4}\right),
$$

$$
\begin{aligned}
S_{3} & =\sum_{\substack{1 \leq l, m \leq R \\
l \neq m, l \neq n, m \neq n}} c_{l} c_{m} a_{l, m} a_{n, l} w_{m} w_{l}=\sum_{\substack{1 \leq l \leq R \\
l \neq n}} a_{n, l} w_{l} \sum_{\substack{1 \leq m \leq R \\
m \neq n, m \neq l}} b_{l, m} w_{m} \\
& =\sum_{\substack{1 \leq l \leq R \\
l \neq n}} a_{n, l} w_{l}\left(\mu v_{l}-b_{l, n} w_{n}\right),
\end{aligned}
$$

and, likewise,

$$
S_{4}=\sum_{\substack{1 \leq m \leq R \\ m \neq n}} a_{n, m} w_{m} \sum_{\substack{1 \leq l \leq R \\ l \neq m, l \neq n}} b_{m, l} w_{l}=\sum_{\substack{1 \leq m \leq R \\ m \neq n}} a_{n, m} w_{m}\left(\mu v_{m}-b_{m, n} w_{n}\right) .
$$

Observing that $S_{3}=S_{4}$, we obtain the formula (20).

Finally, we have by assumption that $B(\boldsymbol{v}+i \boldsymbol{w})=i \mu(\boldsymbol{v}+i \boldsymbol{w})$, so

$$
B \boldsymbol{w}=\mu \boldsymbol{v} \quad \text { and } \quad B \boldsymbol{v}=-\mu \boldsymbol{w} .
$$

Consequently, we have

$$
\mu\|\boldsymbol{w}\|^{2}=\mu \boldsymbol{w}^{T} \boldsymbol{w}=(-B \boldsymbol{v})^{T} \boldsymbol{w}=\left(B^{T} \boldsymbol{v}\right)^{T} \boldsymbol{w}=\boldsymbol{v}^{T} B \boldsymbol{w}=\mu\|\boldsymbol{v}\|^{2},
$$

so for $\mu \neq 0$, we have $\|\boldsymbol{v}\|=\|\boldsymbol{w}\|$.

Finally, let us mention the following nice formula.

Lemma 7. Let $\boldsymbol{u}$ be an eigenvector of $B$ corresponding to the eigenvalue $\mu$. Then

$$
\left|\sum_{1 \leq r \leq R} c_{r} u_{r}\right|^{2}=\sum_{1 \leq r \leq R}\left|c_{r} u_{r}\right|^{2}
$$


Proof. Let $C=\operatorname{diag}\left(c_{1}, \ldots, c_{R}\right)$ and $X=\operatorname{diag}\left(x_{1}, \ldots, x_{R}\right)$. Then

$$
\overline{\boldsymbol{u}}^{T}(X C A C-C A C X) \boldsymbol{u}=\overline{\boldsymbol{u}}^{T} M \boldsymbol{u},
$$

where $m_{r, s}=c_{r} c_{s}$ for $r \neq s$ and 0 otherwise. Therefore,

$$
\overline{\boldsymbol{u}}^{T} M \boldsymbol{u}=\left|\sum_{1 \leq r \leq R} c_{r} u_{r}\right|^{2}-\sum_{1 \leq r \leq R}\left|c_{r} u_{r}\right|^{2} .
$$

On the other hand,

$$
\overline{\boldsymbol{u}}^{T}(X C A C-C A C X) \boldsymbol{u}=\overline{\boldsymbol{u}}^{T}(X B-B X) \boldsymbol{u}=\overline{\boldsymbol{u}}^{T} X i \mu \boldsymbol{u}-i \mu \overline{\boldsymbol{u}}^{T} X \boldsymbol{u}=0,
$$

as $\overline{\boldsymbol{u}}^{T}(-B)=\overline{\boldsymbol{u}}^{T} B^{T}=(B \overline{\boldsymbol{u}})^{T}=(-i \mu \overline{\boldsymbol{u}})^{T}=-i \mu \overline{\boldsymbol{u}}^{T}$. The result follows.

3.4. Back to the spectral norm. Lemma 5 also allows us to deduce the following bounds on the spectral norm of $A(\boldsymbol{x})$.

Corollary 2. $\max _{1 \leq m \leq R} \sum_{1 \leq n \leq R} a_{m, n}^{2} \leq\|A(\boldsymbol{x})\|^{2} \leq 3 \max _{1 \leq m \leq R} \sum_{1 \leq n \leq R} a_{m, n}^{2}$.

Proof. The first inequality is clear, as the $m$-th column of $A$ is the image by $A$ of the $m$-th canonical vector. For the second inequality, we use (16), choosing $n$ such that $\left|u_{n}\right|^{2} \geq\left|u_{m}\right|^{2}$ for all $1 \leq m \leq R$, and $\mu=\|A\|$. We therefore obtain

$$
\|A\|^{2}\left|u_{n}\right|^{2}=\sum_{1 \leq m \leq R} a_{m, n}^{2}\left(\left|u_{m}\right|^{2}+2 \Re\left(u_{n} \bar{u}_{m}\right)\right) \leq \sum_{1 \leq m \leq R} a_{m, n}^{2}\left(\left|u_{m}\right|^{2}+\left|u_{m}\right|^{2}+\left|u_{n}\right|^{2}\right),
$$

so

$$
\|A\|^{2}\left|u_{n}\right|^{2} \leq 3 \sum_{1 \leq m \leq R} a_{m, n}^{2}\left|u_{n}\right|^{2} .
$$

3.5. The classical Hilbert matrix $\boldsymbol{T}_{\boldsymbol{R}}$. The upper bound in Corollary 2 allows us to recover to the original upper bound on $\left\|T_{R}\right\|$, where $T_{R}$ is the Hilbert matrix defined in the introduction:

$$
\left\|T_{R}\right\|^{2} \leq \max _{1 \leq m \leq R} 3 \sum_{\substack{1 \leq n \leq R \\ n \neq m}} \frac{1}{(m-n)^{2}}<3 \cdot 2 \sum_{n \geq 1} \frac{1}{n^{2}}=\pi^{2} .
$$

We now come back to the convergence speed of $\left\|T_{R}\right\|$ toward $\pi$, already mentioned in Section 2. We prove inequality (4), namely that there exist positive constants $a$ and $b$ such that

$$
\frac{a}{R}<\pi-\left\|T_{R}\right\|<\frac{b \log (R)}{R}, \quad \text { where } R \geq 2 .
$$

The lower bound can be deduced from Lemma 5. From (16), we indeed see that if 
$R=2 S+1$, then

$$
\left\|T_{R}\right\|^{2}<6 \sum_{k=1}^{S} \frac{1}{k^{2}}=\pi^{2}-6 \sum_{k>S} \frac{1}{k^{2}}<\pi^{2}-6 \sum_{k>S} \frac{1}{k(k+1)}=\pi^{2}-\frac{6}{S+1},
$$

so

$$
\pi-\left\|T_{R}\right\|>\frac{6}{(S+1)\left(\pi+\left\|T_{R}\right\|\right)}>\frac{3}{\pi(S+1)},
$$

which is of the type $a / R<\pi-\left\|T_{R}\right\|$. Another way to prove this lower bound is to follow the Grenander-Szegő approach of Section 2.2. Let us first recall (6):

$$
\boldsymbol{u}^{*} T_{R} \boldsymbol{u}=\int_{0}^{2 \pi} f(x)|\phi(x)|^{2} d x
$$

where $f(x)=i(x-\pi)$ for $x \in(0,2 \pi)$ and $\phi(x)=\frac{1}{\sqrt{2 \pi}} \sum_{1 \leq n \leq R} u_{n} \exp (i(n-1) x)$, and where $\int_{0}^{2 \pi}|\phi(x)|^{2} d x=\|\boldsymbol{u}\|^{2}=1$. Hence,

$$
\pi-\boldsymbol{u}^{*} i T_{R} \boldsymbol{u}=\int_{0}^{2 \pi} x|\phi(x)|^{2} d x
$$

or, with $E(R)=\left\{\phi(x)=\left.\frac{1}{\sqrt{2 \pi}} \sum_{1 \leq n \leq R} u_{n} \exp (i(n-1) x)\left|\boldsymbol{u} \in \mathbb{C}^{R}, \sum_{1 \leq n \leq R}\right| u_{n}\right|^{2}=1\right\}$,

$$
\pi-\left\|T_{R}\right\|=\inf _{\phi \in E(R)} \int_{0}^{2 \pi} x|\phi(x)|^{2} d x .
$$

It remains to show that the term on the right-hand side of (22) is bounded below by a term of order $1 / R$. To this end, let us consider $\phi \in E(R)$ and $c>0$. Using the Cauchy-Schwarz inequality, we have

$$
\begin{aligned}
\int_{0}^{c}|\phi(x)|^{2} d x & =\frac{1}{2 \pi} \sum_{1 \leq m, n \leq R} u_{m} \bar{u}_{n} \int_{0}^{c} \exp (i(m-n) x) d x \\
& \leq \frac{c}{2 \pi} \sum_{1 \leq m, n \leq R}\left|u_{m}\right|\left|u_{n}\right|=\frac{c}{2 \pi}\left(\sum_{1 \leq n \leq R} 1\left|u_{n}\right|\right)^{2} \\
& \leq \frac{c R}{2 \pi} \sum_{1 \leq n \leq R}\left|u_{n}\right|^{2}=\frac{c R}{2 \pi} .
\end{aligned}
$$

Setting $c=\pi / R$, we obtain $\int_{0}^{\pi / R}|\phi(x)|^{2} d x \leq \frac{1}{2}$. This in turn implies that

$$
\int_{0}^{2 \pi} x|\phi(x)|^{2} d x \geq \int_{\pi / R}^{2 \pi} x|\phi(x)|^{2} d x \geq \frac{\pi}{R} \int_{\pi / R}^{2 \pi}|\phi(x)|^{2} d x \geq \frac{\pi}{2 R}
$$

for all $\phi \in E(R)$, which settles the lower bound in (4). 
To establish the upper bound, we need to find a function $\phi \in E(R)$ such that

$$
\int_{0}^{2 \pi} x|\phi(x)|^{2} d x \leq \frac{b \log R}{R}
$$

for some constant $b>0$. This will indeed ensure the existence of a vector $u-$ namely, the one associated to the function $\phi \in E(R)$ - such that $\left|\boldsymbol{u}^{*} T_{R} \boldsymbol{u}\right| \geq$ $\pi-(b \log R) / R$, thus implying the result.

In view of (23), our goal is to find $\phi \in E(R)$ such that, for $c$ and $\varepsilon$ small,

$$
\int_{c}^{2 \pi}|\phi(x)|^{2} d x \leq \varepsilon
$$

which does imply that

$$
\int_{0}^{2 \pi} x|\phi(x)|^{2} d x \leq c \int_{0}^{c}|\phi(x)|^{2} d x+2 \pi \int_{c}^{2 \pi}|\phi(x)|^{2} d x \leq c+2 \pi \varepsilon .
$$

Let $M$ and $N$ be positive integers such that $N(M-1)+1 \leq R$, and let

The function defined by

$$
g(x)=\left(\sum_{0 \leq m \leq M-1} \exp (\operatorname{imx})\right)^{N} .
$$

$$
\phi(x)=\frac{g(x-c / 2)}{\sqrt{\int_{0}^{2 \pi}|g(x)|^{2} d x}}
$$

belongs to $E(R)$. We claim that, for $M$ and $N$ appropriately chosen, $\phi$ satisfies (24) with both $c$ and $\varepsilon$ small. We first estimate $\int_{0}^{2 \pi}|g(x)|^{2} d x$.

Lemma 8.

$$
\frac{M^{2 N}}{N(M-1)+1} \leq \frac{1}{2 \pi} \int_{0}^{2 \pi}|g(x)|^{2} d x \leq M^{2 N-1} .
$$

Proof. Let $K$ be a positive integer and define the polynomial

$$
P_{K}(t)=\left(\sum_{0 \leq m \leq M-1} t^{m}\right)^{K}=\sum_{0 \leq l \leq K(M-1)} b_{l, K} t^{l} .
$$

Clearly, $b_{l, K}=b_{m, K}$ if $l+m=K(M-1)$. Moreover,

$$
|g(x)|^{2}=\left|P_{N}(\exp (i x))\right|^{2}=\sum_{0 \leq l, m \leq N(M-1)} b_{l, N} b_{m, N} \exp (i(l-m) x),
$$

so

$$
\begin{aligned}
\int_{0}^{2 \pi}|g(x)|^{2} d x & =2 \pi \sum_{0 \leq l \leq N(M-1)} b_{l, N}^{2}=2 \pi \sum_{0 \leq l \leq N(M-1)} b_{l, N} b_{N(M-1)-l, N} \\
& =2 \pi b_{N(M-1), 2 N} .
\end{aligned}
$$


Therefore, what remains to be proven is

$$
\frac{M^{2 N}}{N(M-1)+1} \leq b_{N(M-1), 2 N} \leq M^{2 N-1} .
$$

Using the Cauchy-Schwarz inequality, we obtain

$$
\begin{aligned}
b_{N(M-1), 2 N}=\sum_{0 \leq l \leq N(M-1)} b_{l, N}^{2} \geq \frac{\left(\sum_{0 \leq l \leq N(M-1)} b_{l, N}\right)^{2}}{N(M-1)+1} & =\frac{P_{N}(1)^{2}}{N(M-1)+1} \\
& =\frac{M^{2 N}}{N(M-1)+1} .
\end{aligned}
$$

On the other hand, $P_{2 N}(t)=P_{1}(t) P_{2 N-1}(t)$, so

$$
b_{N(M-1), 2 N}=\sum_{(N-1)(M-1) \leq l \leq N(M-1)} b_{l, 2 N-1} \leq P_{2 N-1}(1) \leq M^{2 N-1},
$$

which completes the proof.

We now set out to prove (24). We retain the same $\phi$ from (26). As a result of the previous lemma, we have

$$
\begin{aligned}
\int_{c}^{2 \pi}|\phi(x)|^{2} d x & \leq \frac{N(M-1)+1}{M^{2 N}} \frac{1}{2 \pi} \int_{c}^{2 \pi}|g(x-c / 2)|^{2} d x \\
& =\frac{N(M-1)+1}{M^{2 N}} \frac{1}{2 \pi} \int_{c / 2}^{2 \pi-c / 2}|g(x)|^{2} d x .
\end{aligned}
$$

Notice that

so

$$
|g(x)|^{2}=\mid\left.\sum_{0 \leq m \leq M-1} \exp (\text { imx } x)\right|^{2 N}=\left(\frac{\sin (M x / 2)}{\sin (x / 2)}\right)^{2 N},
$$

$$
\int_{c / 2}^{2 \pi-c / 2}|g(x)|^{2} d x=2 \int_{c / 2}^{\pi}|g(x)|^{2} d x \leq 2 \int_{c / 2}^{\pi}\left(\frac{\pi \sin (M x / 2)}{x}\right)^{2 N} d x
$$

because $\sin \frac{x}{2} \geq \frac{x}{\pi}$ for $0 \leq x \leq \pi$. This implies

$\int_{c / 2}^{2 \pi-c / 2}|g(x)|^{2} d x \leq 2 \int_{c / 2}^{\infty}\left(\frac{\pi}{x}\right)^{2 N} d x=2 \pi \int_{c / 2 \pi}^{\infty} \frac{1}{y^{2 N}} d y=\frac{2 \pi}{2 N-1}\left(\frac{2 \pi}{c}\right)^{2 N-1}$,

and, correspondingly,

$$
\varepsilon=\int_{c}^{2 \pi}|\phi(x)|^{2} d x \leq \frac{N(M-1)+1}{M^{2 N}} \frac{1}{2 N-1}\left(\frac{2 \pi}{c}\right)^{2 N-1} .
$$

Assuming $R \geq 3$ and defining 


$$
M:=\left\lfloor\frac{2 R}{\log R}\right\rfloor, \quad N:=\left\lfloor\frac{\log R}{2}\right\rfloor, \quad c:=\frac{\pi e \log R}{R}
$$

(where $\lfloor x\rfloor$ denotes the integer part of $x$ ), we verify that $M(N-1)+1 \leq R$ (so $\phi \in E(R)$ ) and prove below that (24) is satisfied with $\varepsilon=O(1 / R)$. Indeed, as $M \geq R / \log R$ and $N(M-1)+1 \leq M(2 N-1)$, we obtain

$$
\frac{N(M-1)+1}{M^{2 N}(2 N-1)(c / 2 \pi)^{2 N-1}}=\left(\frac{c M}{2 \pi}\right)^{1-2 N} \frac{1+N(M-1)}{M(2 N-1)} \leq\left(\frac{c M}{2 \pi}\right)^{1-2 N} \leq e^{1-2 N} \leq \frac{e^{3}}{R},
$$

as $1-2 N<3-\log R$. According to (25), this finally leads to

$$
\int_{0}^{2 \pi} x|\phi(x)|^{2} d x \leq \frac{\pi e \log R}{R}+\frac{2 \pi e^{3}}{R},
$$

which completes the proof of the upper bound in (4). As already mentioned, it has been conjectured in [Preissmann 1985] that of the two bounds in (4), the upper bound is tight. We provide below some numerical simulation data that supports this fact. In Figure 1, the expression

$$
f(R):=\left(\pi-\left\|T_{R}\right\|\right) \frac{R}{\log R}
$$

is represented as a function of $R$, for values of $R$ ranging from 1 to 10,000. Detailed facts can also be established about the eigenvectors of $T_{R}$. In order to ease the notation, suppose that $R=2 S+1$ and that $T_{R}$ is indexed from $-S$ to $S$.

Lemma 9. Let $\boldsymbol{u}$ be an eigenvector of $T_{R}$ corresponding to the eigenvalue $i \mu$, and assume without loss of generality that $u_{0}=1$. For $0 \leq n \leq S$, we have

$$
u_{-n}=-\bar{u}_{n} .
$$

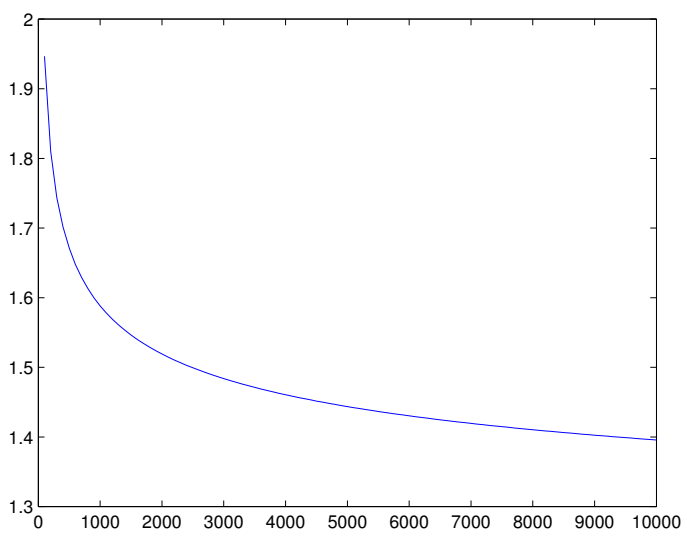

Figure 1. Rescaled gap $f(R)$ between the spectral norm of the infinitedimensional operator $T_{\infty}$ and that of the matrix $T_{R}$, for $1 \leq R \leq 10,000$. 


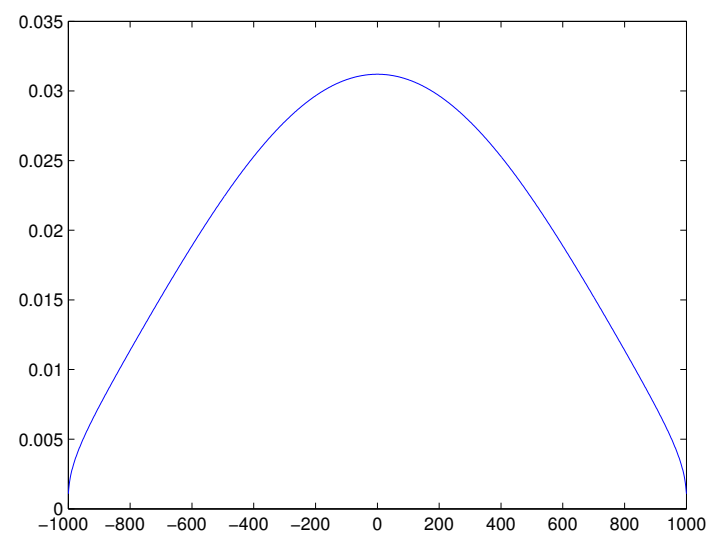

Figure 2. Amplitude $\left\{\left|u_{n}\right|:-R \leq n \leq R\right\}$ of the eigenvector corresponding to the largest eigenvalue of $T_{R}$, with $R=1000$.

Proof. Define $\boldsymbol{v}$ by $v_{n}=-\bar{u}_{-n}$. Then

$$
\left(T_{R} \boldsymbol{v}\right)_{-m}=\sum_{-S \leq n \leq S} \frac{v_{n}}{-m-n}=\sum_{-S \leq n \leq S} \frac{v_{-n}}{-m+n}=-\sum_{-S \leq n \leq S} \frac{v_{-n}}{m-n},
$$

so

$$
\left(T_{R} \boldsymbol{v}\right)_{-m}=\sum_{-S \leq n \leq S} \frac{\bar{u}_{n}}{m-n}=\left(T_{R} \overline{\mathbf{u}}\right)_{m}=(-i \mu \overline{\boldsymbol{u}})_{m}=i \mu v_{-m},
$$

i.e., $\boldsymbol{v}$ is an eigenvector corresponding to the eigenvalue $i \mu$, with $v_{0}=1$. Thus, $\boldsymbol{v}=\boldsymbol{u}$ (as the eigenspace corresponding to $i \mu$ is of dimension 1).

We finally make the following conjecture. Let $\boldsymbol{u}$ be the eigenvector corresponding to the largest eigenvalue $\mu$ in absolute value. Then

$$
\left|u_{m}\right|<\left|u_{n}\right| \text { for all } 0 \leq m<n \leq S .
$$

This conjecture is confirmed numerically; in Figure 2, we represent $\left|u_{n}\right|$ as a function of $n \in\{-S, \ldots, S\}$, for $S=1000$.

From the theoretical point of view, the conjecture also seems reasonable, as $(-1)^{k}\left(T_{R}^{2 k}\right)_{n, n}$ (see Lemma 2) should decrease as $n$ increases (in absolute value). If true, this fact would therefore hold in the limit $k \rightarrow \infty$, which would imply the conjecture on the eigenvector.

\section{References}

[Bombieri et al. 1986] E. Bombieri, J. B. Friedlander, and H. Iwaniec, "Primes in arithmetic progressions to large moduli”, Acta Math. 156:3-4 (1986), 203-251. MR 88b:11058 Zbl 0588.10042

[Choi 1983] M. D. Choi, "Tricks or treats with the Hilbert matrix", Amer. Math. Monthly 90:5 (1983), 
[Grenander and Szegö 1958] U. Grenander and G. Szegö, Toeplitz forms and their applications, University of California Press, Berkeley, 1958. Reprinted Chelsea, New York, 1984. MR 20 \#1349 Zbl 0080.09501

[Hardy et al. 1952] G. H. Hardy, J. E. Littlewood, and G. Pólya, Inequalities, 2nd ed., Cambridge University Press, 1952. MR 13,727e Zbl 0047.05302

[Laeng 2007] E. Laeng, "Remarks on the Hilbert transform and on some families of multiplier operators related to it”, Collect. Math. 58:1 (2007), 25-44. MR 2008b:42012 Zbl 1135.42006

[Laeng 2009] E. Laeng, "Sharp norm inequalities for the truncated Hilbert transform", J. Math. Inequal. 3:1 (2009), 123-127. MR 2010a:42024 Zbl 1158.42004

[Matthews 2002] K. Matthews, "Hilbert's inequality", unpublished notes, 2002, available at http:// www.numbertheory.org/pdfs/hilbert.pdf.

[Montgomery and Vaughan 1973] H. L. Montgomery and R. C. Vaughan, "The large sieve", Mathematika 20 (1973), 119-134. MR 51 \#10260 Zbl 0296.10023

[Montgomery and Vaughan 1974] H. L. Montgomery and R. C. Vaughan, "Hilbert's inequality", J. London Math. Soc. (2) 8 (1974), 73-82. MR 49 \#2544 Zbl 0281.10021

[Preissmann 1984] E. Preissmann, "Sur une inégalité de Montgomery-Vaughan”, Enseign. Math. (2) 30:1-2 (1984), 95-113. MR 85e:11067 Zbl 0548.10031

[Preissmann 1985] E. Preissmann, Autour des inégalités de Hilbert-Montgomery-Vaughan, Ph.D. thesis, University of Lausanne, 1985.

[Preissmann 1987] E. Preissmann, "Équations fonctionnelles et estimations de normes de matrices", Aequationes Math. 32:2-3 (1987), 195-212. MR 88i:39014 Zbl 0625.39009

[Slepian 1978] D. Slepian, "Prolate spheroidal wave functions, Fourier analysis and uncertainity, V: The discrete case”, Bell System Tech. J. 57:5 (1978), 1371-1430. Zbl 0378.33006

[Titchmarsh 1926] E. C. Titchmarsh, "Reciprocal formulae involving series and integrals", Math. Z. 25:1 (1926), 321-347. MR 1544814 JFM 52.0213.03

[Varah 1993] J. M. Varah, “The prolate matrix", Linear Algebra Appl. 187 (1993), 269-278. MR 94e:15058 Zbl 0782.15014

[Wilf 1970] H. S. Wilf, Finite sections of some classical inequalities, Ergebnisse der Mathematik und ihrer Grenzgebiete 52, Springer, New York, 1970. MR 42 \#6643 Zbl 0199.38301

Received August 17, 2012.

\section{EMMANUEL PREISSMANN}

Swiss Federal Institute of Technology

CH-1015 LAUSANNE

SWITZERLAND

emmanuel.preissmann@gmail.com

\section{OLIVIER LÉVÊQUE}

Swiss Federal Institute of Technology

CH-1015 LAUSANNE

SWITZERLAND

olivier.leveque@epfl.ch 


\title{
PACIFIC JOURNAL OF MATHEMATICS
}

\author{
msp.org/pjm
}

Founded in 1951 by E. F. Beckenbach (1906-1982) and F. Wolf (1904-1989)

\section{EDITORS}

V. S. Varadarajan (Managing Editor)

Department of Mathematics

University of California

Los Angeles, CA 90095-1555

pacific@math.ucla.edu

Paul Balmer

Department of Mathematics

University of California

Los Angeles, CA 90095-1555

balmer@math.ucla.edu

Daryl Cooper

Department of Mathematics

University of California

Santa Barbara, CA 93106-3080 cooper@math.ucsb.edu

Jiang-Hua $\mathrm{Lu}$

Department of Mathematics

Pokfulam Rd., Hong Kong jhlu@maths.hku.hk
The University of Hong Kong

Don Blasius

Department of Mathematics University of California

Los Angeles, CA 90095-1555

blasius@math.ucla.edu

Robert Finn

Department of Mathematics Stanford University

Stanford, CA 94305-2125

finn@math.stanford.edu

Sorin Popa

Department of Mathematics

University of California

Los Angeles, CA 90095-1555 popa@math.ucla.edu

Paul Yang

Department of Mathematics Princeton University

Princeton NJ 08544-1000

yang@math.princeton.edu

\section{PRODUCTION}

Silvio Levy, Scientific Editor, production@msp.org

\section{SUPPORTING INSTITUTIONS}

ACADEMIA SINICA, TAIPEI

CALIFORNIA INST. OF TECHNOLOGY

INST. DE MATEMÁTICA PURA E APLICADA

KEIO UNIVERSITY

MATH. SCIENCES RESEARCH INSTITUTE

NEW MEXICO STATE UNIV.

OREGON STATE UNIV.

\author{
STANFORD UNIVERSITY \\ UNIV. OF BRITISH COLUMBIA \\ UNIV. OF CALIFORNIA, BERKELEY \\ UNIV. OF CALIFORNIA, DAVIS \\ UNIV. OF CALIFORNIA, LOS ANGELES \\ UNIV. OF CALIFORNIA, RIVERSIDE \\ UNIV. OF CALIFORNIA, SAN DIEGO \\ UNIV. OF CALIF., SANTA BARBARA
}

\author{
Vyjayanthi Chari \\ Department of Mathematics \\ University of California \\ Riverside, CA 92521-0135 \\ chari@math.ucr.edu \\ Kefeng Liu \\ Department of Mathematics \\ University of California \\ Los Angeles, CA 90095-1555 \\ liu@math.ucla.edu \\ Jie Qing \\ Department of Mathematics \\ University of California \\ Santa Cruz, CA 95064 \\ qing@cats.ucsc.edu
}

These supporting institutions contribute to the cost of publication of this Journal, but they are not owners or publishers and have no responsibility for its contents or policies.

See inside back cover or msp.org/pjm for submission instructions.

The subscription price for 2013 is US \$400/year for the electronic version, and \$485/year for print and electronic.

Subscriptions, requests for back issues and changes of subscribers address should be sent to Pacific Journal of Mathematics, P.O. Box 4163, Berkeley, CA 94704-0163, U.S.A. The Pacific Journal of Mathematics is indexed by Mathematical Reviews, Zentralblatt MATH, PASCAL CNRS Index, Referativnyi Zhurnal, Current Mathematical Publications and the Science Citation Index.

The Pacific Journal of Mathematics (ISSN 0030-8730) at the University of California, c/o Department of Mathematics, 798 Evans Hall \#3840, Berkeley, CA 94720-3840, is published twelve times a year. Periodical rate postage paid at Berkeley, CA 94704, and additional mailing offices. POSTMASTER: send address changes to Pacific Journal of Mathematics, P.O. Box 4163, Berkeley, CA 94704-0163.

PJM peer review and production are managed by EditFLOW ${ }^{\circledR}$ from Mathematical Sciences Publishers.

\section{PUBLISHED BY}

mathematical sciences publishers

nonprofit scientific publishing

http://msp.org/

(C) 2013 Mathematical Sciences Publishers 


\section{PACIFIC JOURNAL OF MATHEMATICS}

Volume $265 \quad$ No. $1 \quad$ September 2013

Genus-two Goeritz groups of lens spaces

SANGBUM CHO

A compact embedding theorem for generalized Sobolev spaces

SENG-KeE ChUa, SCOTT Rodney and Richard L. WheEden

Partial integrability of almost complex structures and the existence of

solutions for quasilinear Cauchy-Riemann equations

CHONG-KYU HAN and JONG-DO PARK

An overdetermined problem in potential theory

DMITRY KHAVINSON, ERIK LUNDBERG and RAZVAN TEODORESCU

Quasisymmetric homeomorphisms on reducible Carnot groups

XIANGDONG XIE

Capillarity and Archimedes' principle

JOHN MCCUAN and RAY TREINEN

Generalized eigenvalue problems of nonhomogeneous elliptic operators and their application

DUmitru Motreanu and Mieko TANaKa

Weighted Ricci curvature estimates for Hilbert and Funk geometries

SHIN-ICHI OHTA

On generalized weighted Hilbert matrices

EMMANUEL PREISSMANN and OLIVIER LÉVÊQUE

Unique prime decomposition results for factors coming from wreath product 221 groups

J. OWEN SizEMORE and ADAM WinCHESTER

On volume growth of gradient steady Ricci solitons

Guofang Wei and Peng Wu

Classification of moduli spaces of arrangements of nine projective lines

FEI YE 